\title{
Effect of Resveratrol on Oral Cancer Cell Invasion Induced by Lysophosphatidic Acid
}

\author{
Jin Young Kim, Kyung Hwa Cho, and Hoi Young Lee ${ }^{\dagger}$ \\ Department of Pharmacology, College of Medicine, Konyang University, Daejeon 35365, Korea
}

\begin{abstract}
The aim of the current study was to demonstrate the potential therapeutic efficacy of resveratrol in oral cancer patients. Lysophosphatidic acid (LPA) intensifies cancer cell invasion and metastasis, whereas resveratrol, a natural polyphenolic compound, possesses antitumor activity, suppressing cell proliferation and progression in various cancer cell lines (ovarian, gastric, oral, pancreatic, colon, and prostate cancer cells). In addition, resveratrol has been identified as an inhibitor of LPA-induced proteolytic enzyme expression and ovarian cancer invasion. Furthermore, resveratrol was shown to inhibit oral cancer cell invasion by downregulating hypoxia-inducible factor $1 \alpha$ and vascular endothelial growth factor expression. Recently, we demonstrated that LPA is important for the expression of transcription factors TWIST and SLUG during epithelial-mesenchymal transition (EMT) in oral squamous carcinoma cells. In this study, we treated serum-starved cultures of oral squamous carcinoma cell line YD-10B with resveratrol for 24 hours prior to stimulation with LPA. To identify an optimal resveratrol concentration that does not induce apoptosis in oral squamous carcinoma cells, we determined the toxicity of resveratrol in YD-10B cells by assessing their viability using the MTT assay. Another assay was performed using Matrigel-coated cell culture inserts to detect oral cancer cell invasion activity. Immunoblotting was applied for analyzing protein expression of SLUG, TWIST1, E-cadherin, and GAPDH. We demonstrated that resveratrol efficiently inhibited LPA-induced oral cancer cell EMT and invasion by downregulating SLUG and TWIST1 expression. Therefore, resveratrol may potentially reduce oral squamous carcinoma cell invasion and metastasis in oral cancer patients, improving their survival outcomes. In summary, we identified new targets for the development of therapies against oral cancer progression and characterized the therapeutic potential of resveratrol for the treatment of oral cancer patients.
\end{abstract}

Key Words: Epithelial-mesenchymal transition, Lysophospholipids, Mouth neoplasms, Stilbenes

\section{Introduction}

The World Health Organization (WHO) defines oral cancer as a group of malignant tumors that occur in the oral and pharyngeal regions, which include the lips, salivary glands, tongue, gingiva, surrounding periodontal tissue, oral floor, soft palate, hard palate, buccal mucosal, vestibular gingiva, tonsil, oropharyngeal region, and hypopharyngeal region ${ }^{1)}$. Squamous cell carcinoma, which affects the squamous epithelium comprising the oral mucosa, accounts for $90 \%$ of all cases of oral cancer. Oral cancer is highly lethal, and the survival rate at five or more years after the cancer diagnosis is below $60 \%^{2)}$. Each year, 529,000 new oral cancer cases are diagnosed, and more than 300,000 people die from it. The major progression event in these cancers is the metastasis of a malignant tumor from the primary site to other organs ${ }^{3)}$. Patients with invasive oral cancer have a poor prognosis, with less than $10 \%$ chance of survival.

Previous studies revealed that the epithelialmesenchymal transition (EMT) plays an important role during the invasion of various cancer types. During EMT, epithelial cells lose their polarity and cell-to-cell adhesion to become mesenchymal stem cells. The EMT is a crucial process in the development of invasive diseases such as cancer that is closely associated with cell adhesion, migration, and invasion ${ }^{4)}$. Major EMT factors include TWIST, SLUG, Snail, and E-cadherin ${ }^{4}$. 
Previous studies have investigated the effect of various natural products on metastasis and invasion, and they are still actively pursued. Resveratrol, a polyphenolic phytoalexin, is typically found in grapes, peanuts, and strawberries. Following the formulation of the French Paradox concept, which refers to the observation that red wine reduces deaths associated with cardiovascular diseases among the populations of France or Greece, researchers have begun to focus on resveratrol, a substance found in red wine ${ }^{5)}$.

Resveratrol reportedly possesses anti-platelet and antioxidant activities, inhibits low-density lipoprotein oxidase activity, protects the cardiovascular system, and prevents other diseases ${ }^{6}$. It reportedly exhibited excellent in vitro and in vivo antitumor activity ${ }^{7-11)}$, induced cell apoptosis in oral cancer via various signaling pathways, and mediated cell cycle arrest ${ }^{12-15}$ ).

In our previous study, we found that lysophosphatidic acid (LPA), which promotes cancer cell migration and growth, and increases cancer cell survival, reduces the expression of E-cadherin, an EMT marker, and increases the expression of TWIST and SLUG, and, ultimately, increases oral cancer cell invasion. In this study, we hypothesized that resveratrol may interfere with the LPA-induced expression of TWIST and SLUG.

\section{Materials and Methods}

\section{Cell culture}

YD-10B oral squamous carcinoma cells were obtained from the Department of Oral Pathology, School of Dentistry, Yonsei University. They were suspended on a T75 plate in RPMI 1640, containing $10 \%$ fetal bovine serum (FBS) (Hyclone, Logan, UT, USA) and 1\% penicillin/1\% streptomycin (Lonza, Allendale, NJ, USA), and cultured inside an incubator at $5 \% \mathrm{CO}_{2}$ and $37^{\circ} \mathrm{C}$. After cells reached $80 \%$ confluency, they were washed twice in phosphate buffered saline solution (PBS) and subcultured in the presence of trypsin and ethylenediaminetetraacetic acid (EDTA; Lonza, Basel, Switzerland).

\section{Cell survival analysis}

Cells were cultured at $1 \times 10^{4}$ cells per well in a 96-well plate prior to being subjected to a 12-hour serum starvation in RPMI lacking FBS. Then, cells were treated with resveratrol (Sigma-Aldrich, St. Louis, MO, USA) at different concentrations for 24 hours. Following two washes with PBS, MTT ([3,4,5,-dimethylthiazol-2yl]-5diphenyltetrazolium bromide) was added at $0.5 \mathrm{mg} / \mathrm{ml}$ to the wells, and incubation was continued at $5 \% \mathrm{CO}_{2}$ and $37^{\circ} \mathrm{C}$ for two hours. After two washes with PBS, $100 \mu \mathrm{l}$ of dimethyl sulfoxide (DMSO; Sigma-Aldrich) was added to each well. Optical density was measured using an ELISA plate reader (BioTek Instruments, Winooski, VT, USA) at $540 \mathrm{~nm}$.

\section{Immunoblotting}

Following two washes with PBS, a radioimmunoprecipitation (RIPA) buffer was added to the cells. Cell proteins were scraped off the plates and incubated at $4{ }^{\circ} \mathrm{C}$ for 10 minutes. Then, cells were centrifuged at $4^{\circ} \mathrm{C}$ and $13,000 \mathrm{rpm}$ for 15 minutes. Next, only the protein fraction was collected and quantified using a BCA protein assay reagent (Thermo Fisher Scientific, Waltham, MA, USA). In total, $50 \mu \mathrm{g}$ of the quantified proteins were separated according to their size by $8 \%$ or $12 \%$ SDS-polyacrylamide gel electrophoresis (PAGE) and transferred to polyvinylidene difluoride (PVDF) membranes. On a shaker, the membranes were incubated in EzBlockChemi (ATTO, Tokyo, Japan), which had been diluted by $1 / 5$ with a saline solution, for 30 minutes, and exposed to primary antibodies in a refrigerator at $4^{\circ} \mathrm{C}$ for 16 hours. Following the incubation with the primary antibodies, the membranes were washed twice with TBST $(0.1 \%$ Tween 20 in Tris buffered saline [TBS]), and incubated with secondary antibodies for 2 hours. Then, the membranes were washed twice with TBST, and immunoreactive bands were detected by ImageQuant 300 (GE Healthcare, Little Chalfont, UK) using ECL (Pierce, Rockford, IL, USA). The primary antibodies were TWIST1, GAPDH (Santa Cruz Biotechnology, Dallas, TX, USA), SLUG (Cell Signaling Technology, Danvers, MA, USA), and E-cadherin (BD Biosciences, San Jose, CA, USA).

\section{In vitro invasion assay}

An in vitro invasion assay was performed using 
membranes coated with Matrigel (BD Biosciences) in a modified Boyden chamber (Neuro Pro, Gaithersburg, MD, USA). Following a 12-hour culture at $37^{\circ} \mathrm{C}$ inside an incubator, the membranes were fixed and stained with Diff-Quik (Dade Behring, Newark, DE, USA). The numbers of invading cells quantitated by counting the number of cells in six random high-power fields for each replicate $(200 \times)$ with a light microscope. The results are from the experiment in triplicate of three independent experiments.

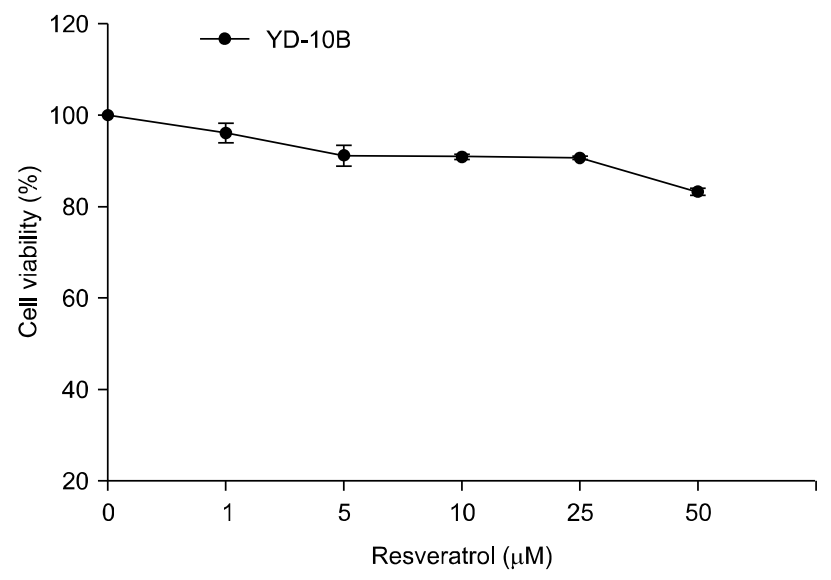

Fig. 1. The viability of oral cancer cell was analyzed by MTT assays. Cell viability experiments were from the experiment in triplicate of three independent experiments. Oral cancer YD-10B cells were survived over $90 \%$ when they are treated with $25 \mu \mathrm{M}$ of resveratrol.

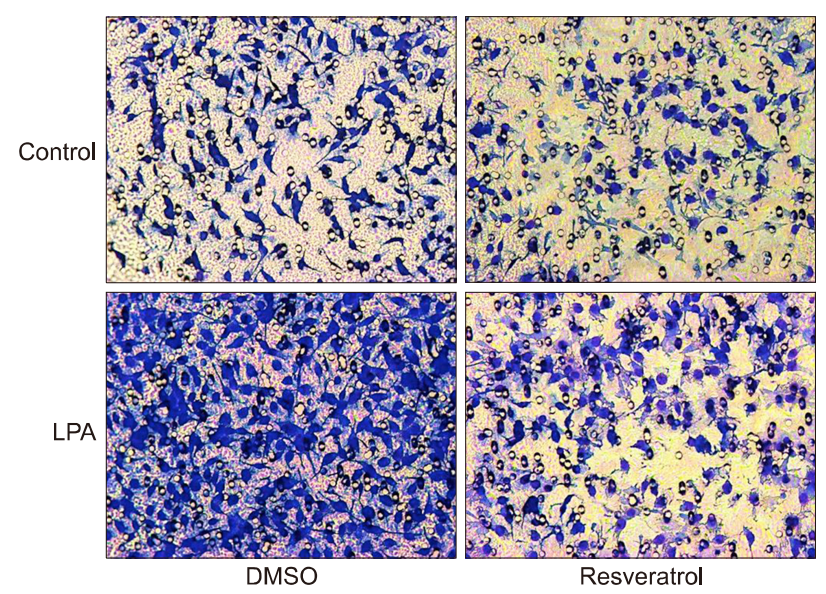

\section{Statistical analysis}

All collected data were analyzed using IBM SPSS Statistics ver. 22.0 (IBM, Armonk, NY, USA), with the level of statistical significance set at $5 \%$. In vitro invasion assay p-value obtained from the paired t-test. Value are presented as mean \pm standard deviation ( $\mathrm{p}<0.01$ vs. control, $\mathrm{p}<0.01$ vs. control and LPA).

\section{Results}

1. Oral cancer YD-10B cell survival rate with resveratrol concentrations

To investigate the optimal resveratrol concentration at which cell apoptosis is not induced in oral cancer cells, a cell survival analysis was performed. As shown in Fig. 1, over $90 \%$ of the cells were survived when cells were treated with resveratrol at $25 \mu \mathrm{M}$. Based on these results, a resveratrol concentration of $25 \mu \mathrm{M}$ was used in experiments shown in Fig. 2 and 3.

\section{Inhibition of oral cancer cell invasion by resveratrol}

A modified Boyden chamber assay was performed to investigate the effect of resveratrol on oral cancer cell invasion induced by LPA in YD-10B cells. As shown in Fig. 2, oral cancer cell invasion was significantly increased in YD-10B cells treated with LPA. In contrast, resveratrol profoundly reduced LPA-induced oral cancer

Fig. 2. Resveratrol inhibits lysophosphatidic acid (LPA)-induced oral cancer cell invasion. The serum-starved YD-10B cells were pretreated with resveratrol for 1 hour and in vitro invasion were analyzed against LPA (mean \pm standard deviation; $* \star p<0.01$ vs. control, $\# \#<0.01$ vs. control and LPA). Cell invasion experiments were from the experiment in triplicate of three independent experiments. This results show that resveratrol reduce LPA-induced invasion of oral cancer cells. DMSO: dimethyl sulfoxide. 




Fig. 3. Resveratrol reduces Iysophosphatidic acid (LPA)-induced oral cancer epithelial-mesenchymal transition (EMT). Representative blots from three independent experiments with similar results. The present data indicate that resveratrol significantly inhibits the expression of LPA-induced EMT factors (TWIST and Slug) in oral cancer cells. DMSO: dimethyl sulfoxide.

cell invasion compared to control. These results demonstrated that resveratrol can reduce LPA-induced oral cancer cell invasion.

\section{Inhibition of LPA-induced expression of TWIST and SLUG by resveratrol}

Immunoblotting was performed to determine if resveratrol affects the protein expression of major EMT transcription factors involved in oral cancer cell invasion. As shown in Fig. 3, resveratrol significantly inhibited the LPA-induce expression of TWIST and SLUG. On the other hand, the expression of E-cadherin downregulated by LPA was completely restored by resveratrol. These results show that resveratrol inhibits the LPA-induced expression of EMT factors, leading to attenuation of oral cancer cell EMT and invasion.

\section{Discussion}

A high incidence rate of oral cancer has been reported worldwide since the 19th century, and the WHO predicts future increases in the incidence rate. Therefore, oral cancer has been identified as a major health issue ${ }^{2,16,17)}$. The causes of oral cancer include smoking, high alcohol consumption, and human papillomavirus infection ${ }^{18-20)}$. Known internal cellular factors include molecular changes such as the activation of Notch, affecting cell-cycle and mitogenic signaling, and the inactivation of p53 tumor suppressor $^{21,22)}$.

LPA is formed extracellularly by autotaxin, a secreted enzyme, that removes the choline group from lysophosphatidylcholine. LPA is known to induce cell fibrosis via six types of $\mathrm{G}$ protein-coupled receptors that are associated with the induction of liver cirrhosis and pulmonary fibrosis ${ }^{23)}$. In addition, LPA also promotes cell cancer proliferation and activates of various signaling pathways to accelerate cell cycle progression ${ }^{23}$. Furthermore, a recent study reported that LPA augments the migration of oral cancer cells through $\mathrm{G}$ protein-coupled LPA receptor $3^{24}$. Our research team observed that LPA induces the expression of TWIST and SLUG, which are major EMT transcription factors, to aggravate oral cancer cell invasion ${ }^{25)}$. Our results suggested that LPA downregulates E-cadherin expression and promoted oral cancer cell migration and invasion through upregulation of EMT transcription factors, TWIST and SLUG. Because LPA induces oral cancer cell migration and infiltration, the increased expression levels of autotaxin, LPA receptor 3, and EMT transcription factors may be used as biomarkers for oral cancer diagnosis and treatment.

Resveratrol, which is a natural polyphenol, inhibits hypoxia-inducible factor $1 \alpha$ accumulation ${ }^{26)}$ and reduces the expression of vascular endothelial growth factor, an inducer of angiogenesis ${ }^{27)}$, thereby reducing oral cancer cell invasion. In this study, we observed that LPA, which is known to stimulate oral cancer cell migration and invasion, increases the expression of two major EMT transcription factors, TWIST and SLUG that were effectively downregulated by resveratrol. Therefore, resveratrol may be useful in the treatment of patients with oral cancer. The effects of resveratrol may be enhanced by concomitant administration of resveratrol with platinumbased chemotherapeutic agents used in existing oral cancer treatments. In conclusion, we hope that our findings will stimulate the interest in testing resveratrol as a potential medication for reducing oral cancer cell migration and invasion. 


\section{Acknowledgements}

This study was supported by a grant from the Basic Science Research Program through the National Research Foundation of Korea (NRF) funded by the Ministry of Education, Science and Technology (NRF-2017R1E1A1 A01074091).

\section{References}

1. Robinson KL, Macfarlane GJ: Oropharyngeal cancer incidence and mortality in Scotland: are rates still increasing? Oral Oncol 39: 31-36, 2003. https://doi.org/10.1016/S1368-8375(02)00014-3

2. Han JH, Kim EK, Lim SH, Kim CH: Literature review on the incidence and risk factor of oral cancer. J Dent Hyg Sci 12: 451-458, 2012.

3. Roepman P, Wessels LF, Kettelarij N, et al.: An expression profile for diagnosis of lymph node metastases from primary head and neck squamous cell carcinomas. Nat Genet 37: 182-186, 2005. https://doi.org/10.1038/ng1502

4. Mandal M, Myers JN, Lippman SM, et al.: Epithelial to mesenchymal transition in head and neck squamous carcinoma: association of Src activation with E-cadherin down-regulation, vimentin expression, and aggressive tumor features. Cancer 112: 2088-2100, 2008. https://doi.org/10.1002/cncr.23410

5. Kopp P: Resveratrol, a phytoestrogen found in red wine. A possible explanation for the conundrum of the 'French paradox'? Eur J Endocrinol 138: 619-620, 1998. https://doi.org/10.1530/eje.0.1380619

6. Savouret JF, Quesne M: Resveratrol and cancer: a review. Biomed Pharmacother 56: 84-87, 2002. https://doi.org/10.1016/S0753-3322(01)00158-5

7. Gupta SC, Kannappan R, Reuter S, Kim JH, Aggarwal BB: Chemosensitization of tumors by resveratrol. Ann N Y Acad Sci 1215: 150-160, 2011. https://doi.org/10.1111/j.1749-6632.2010.05852.x

8. Tyagi A, Singh RP, Agarwal C, Siriwardana S, Sclafani RA, Agarwal R: Resveratrol causes Cdc2-tyr15 phosphorylation via ATM/ATR-Chk1/2-Cdc25C pathway as a central mechanism for $\mathrm{S}$ phase arrest in human ovarian carcinoma Ovcar-3 cells. Carcinogenesis 26: 1978-1987, 2005. https://doi.org/10.1093/carcin/bgi165

9. Yang SH, Kim JS, Oh TJ, et al.: Genome-scale analysis of resveratrol-induced gene expression profile in human ovarian cancer cells using a cDNA microarray. Int $\mathrm{J}$ Oncol 22: 741-750, 2003. https://doi.org/10.3892/ijo.22.4.741

10. Kim YN, Choe SR, Cho KH, et al.: Resveratrol suppresses breast cancer cell invasion by inactivating a RhoA/YAP signaling axis. Exp Mol Med 49: e296, 2017. https://doi.org/10.1038/emm.2016.151

11. Kim SH, Cho KH, Kim YN, et al.: Resveratrol attenuates norepinephrine-induced ovarian cancer invasiveness through downregulating hTERT expression. Arch Pharm Res 39: 240-248, 2016. https://doi.org/10.1007/s12272-015-0666-8

12. Kim SE, Shin SH, Lee JY, et al.: Resveratrol induces mitochondrial apoptosis and inhibits epithelial-mesenchymal transition in oral squamous cell carcinoma cells. Nutr Cancer 70: 125-135, 2018.

https://doi.org/10.1080/01635581.2018.1397708

13. Chang $\mathrm{CH}$, Lee $\mathrm{CY}$, Lu CC, et al.: Resveratrol-induced autophagy and apoptosis in cisplatin-resistant human oral cancer CAR cells: a key role of AMPK and Akt/mTOR signaling. Int J Oncol 50: 873-882, 2017.

https://doi.org/10.3892/ijo.2017.3866

14. Baek SH, Ko JH, Lee H, et al.: Resveratrol inhibits STAT3 signaling pathway through the induction of SOCS-1: role in apoptosis induction and radiosensitization in head and neck tumor cells. Phytomedicine 23: 566-577, 2016. https://doi.org/10.1016/j.phymed.2016.02.011

15. Yu XD, Yang JL, Zhangddd, et al.: Resveratrol inhibits oral squamous cell carcinoma through induction of apoptosis and G2/M phase cell cycle arrest. Tumour Biol 37: 2871-2877, 2016. https://doi.org/10.1007/s13277-015-3793-4

16. Subramanian S, Sankaranarayanan R, Bapat B, et al.: Cost-effectiveness of oral cancer screening: results from a cluster randomized controlled trial in India. Bull World Health Organ 87: 200-206, 2009. https://doi.org/10.2471/BLT.08.053231

17. Kim HD, Paik DI, Jung SH, Park DY, Kim JB: Factors influencing knowledge about early signs and causes of oral cancer in Korean elder. J Korean Acad Dent Health 28: 570-582, 2004.

18. Lee EJ, Kim J, Lee SA, et al.: Characterization of newly established oral cancer cell lines derived from six squamous 
cell carcinoma and two mucoepidermoid carcinoma cells. Exp Mol Med 37: 379-390, 2005.

https://doi.org/10.1038/emm.2005.48

19. Ahrendt SA, Chow JT, Yang SC, et al.: Alcohol consumption and cigarette smoking increase the frequency of $\mathrm{p} 53$ mutations in non-small cell lung cancer. Cancer Res 60: 3155-3159, 2000.

20. Balaram P, Sridhar H, Rajkumar T, et al.: Oral cancer in southern India: the influence of smoking, drinking, paan-chewing and oral hygiene. Int J Cancer 98: 440-445, 2002. https://doi.org/10.1002/ijc. 10200

21. Chen SJ, Liu H, Liao CT, et al.: Ultra-deep targeted sequencing of advanced oral squamous cell carcinoma identifies a mutation-based prognostic gene signature. Oncotarget 6: 18066-18080, 2015. https://doi.org/10.18632/oncotarget.3768

22. Tamura T, Ichikawa T, Nakahata S, et al.: Loss of NDRG2 expression confers oral squamous cell carcinoma with enhanced metastatic potential. Cancer Res 77: 2363-2374, 2017. https://doi.org/10.1158/0008-5472.CAN-16-2114

23. Benesch MG, Ko YM, McMullen TP, Brindley DN:
Autotaxin in the crosshairs: taking aim at cancer and other inflammatory conditions. FEBS Lett 588: 2712-2727, 2014. https://doi.org/10.1016/j.febslet.2014.02.009

24. Brusevold IJ, Tveteraas IH, Aasrum M, Ødegård J, Sandnes DL, Christoffersen T: Role of LPAR3, PKC and EGFR in LPA-induced cell migration in oral squamous carcinoma cells. BMC Cancer 14: 432, 2014.

https://doi.org/10.1186/1471-2407-14-432

25. Cho KH: Lysophosphatidic acid-induced TWIST1 and slug expression in oral cancer cell invasion. J Dent Hyg Sci 17: 433-438, 2017. https://doi.org/10.17135/jdhs.2017.17.5.433

26. Shan Z, Yang G, Xiang W, Pei-jun W, Bin Z: Effects of resveratrol on oral squamous cell carcinoma (OSCC) cells in vitro. J Cancer Res Clin Oncol 140: 371-374, 2014. https://doi.org/10.1007/s00432-013-1575-1

27. Zhang Q, Tang X, Lu QY, Zhang ZF, Brown J, Le AD: Resveratrol inhibits hypoxia-induced accumulation of hypoxia-inducible factor-1alpha and VEGF expression in human tongue squamous cell carcinoma and hepatoma cells. Mol Cancer Ther 4: 1465-1474, 2005. https://doi.org/10.1158/1535-7163.MCT-05-0198 